contained structured questions of the multiple-choice type, but a certain number offered the possibility for additional comments; open-ended questions were also included.

Results: Answers from the 66 respondents indicated that 53 persons $(80.3 \%)$ had been subjected to threats and/or violence. This was experienced by the majority as unpleasant to some extent or other. Moreover, the patient-paramedic relationship is affected as soon as the paramedic is exposed to threat or the use of violence.

Conclusion: Threats and violence are a reality in the provision of ambulance service, and measures must be taken so that the effects on caregivers as well as patients are allayed. Keywords: ambulance providers; emergency medical care; paramedics; patients; threats; violence

Prebosp Disast Med 2002;17(s2):s84-85.

\section{Utilization of Communication Technology to Improve Team Recall Efficiency}

Ming-Che Tsai; Chia-Chang Chuang; Chib-Hsien Chi The Executive Office of South NDAMT, National Cheng Kung University Hospital, Tainan, Taiwan ROC

Background: The Disaster Medical Assistance Team (DMAT) in Taiwan was organized through voluntary individual hospital participation starting in 2000. At present, there are two levels of DMATs (national vs. local) made up of 60 and 20 people, respectively, as basic operation modules. The National DMAT of the south consisted of six local DMATs. Efficient communication (vertical vs. horizontal) during the time of recall as well as team status update, warrants opportune response and deployment.

Methods: A voice message center was set up on a GSM mobile phone system in a preregistered call list. For a deployment order, the executive office only has to send one voice message by predetermined priorities such as individual mobile phone first, followed by work phones, and then home phone. A pilot test was carried out from 11 to 21 November 2002. Horizontal contacts among 13 team leaders (Group I), and 18 team members in one operation unit (Group II) were processed vertically.

Results: The average response times in Groups I and II were 5.2 and 4.9 minutes ( $\mathrm{min}$ ), respectively; the $90 \%$ fractile response time was 14.3 and $11.7 \mathrm{~min}$. The percentage of calls received and confirmed by reply in Group I was $67.1 \%$, and in Group II was $61.4 \%$. To increase the overall coverage rate, a tiered call-out system was supplemented by written message via mobile and fax.

Conclusion: To improve communication management, it's important to modify message delivery. Message contents were categorized into emergency, urgent, and routine notification. Emergency recall will be made by voice message, urgent communication by written message, and routine by fax. In general, this modified recall system with the advancement of communication technology can serve as a DMAT quality indicator. Periodic auditing will strengthen the chain of command, and also prompt the recall process to provide a better disaster response.

Keywords: communication; medical assistance team (DMAT), disaster management; messages; quality; recall; response times; technology; voice message center

Prebosp Disast Med 2002;17(s2):s85

Medical Assistance in International Media Center

\section{during 2002 FIFA World Cup Soccer Games in} South Korea

Soon-Joo Wang; ${ }^{1}$ Hyoung-Gon Song ${ }^{2}$

1. Hallym University Sacred Heart Hospital, South Korea

2. Samsung Medical Center, Sungkyunkwan University School of Medicine, South Korea

Introduction: The International Media Center (IMC) was made up of an International Broadcast Center (IBC) and a Main Press Center (MPC) for the 2002 FIFA World Cup Soccer Games. It was established and maintained in Seoul, South Korea, and covered an area of 40,000 square meters, which served approximately 12,000 press personnel and related persons. A medical-aid station was established in the IMC and operated for 45 days during the World Cup competitions. This presentation provides a description of characteristics and medical data obtained in the IMC medical-aid station.

Methods: Data were abstracted from preformed, special medical records of IMC medical aid. Some of the variables collected and analyzed included: (1) Total numbers of patients; (2) whether a first visit or revisit; (3) simple drug requirements; (4) native or foreigner to South Korea; (5) chief complaint; (6) disease or injury; (7) proportions of treatments by class; and (8) courses after medical care. Data also were analyzed for serial change by week, patterns according to characteristics of games, and for days with or without games.

Results: 1,000 patients visited, with an average 22 patients per day; $68 \%$ of patients visited the station for the first time, and $59 \%$ were foreigners. There was no serial increase of numbers or the rate of patient visits except for revisiting patients. There were more males than females overall (except for patients in the second decade of age); and all of the patients over 60 years of age were male. Sore throat was the characteristic chief complaint of patients working in IBC. The rate of patients injured was $19.6 \%$, and the maximal number of patients injured were males in their fifties. Of all patients, $89 \%$ were discharged, and $2.3 \%$ were transferred. Among treatments, excluding those requiring medication, $11 \%$ required dressings. $73.9 \%$ of patients received oral medication, and $10.0 \%$ required injections.

Conclusion: Medical assistance for media centers in large sports events have different characteristics compared with medical assistance required for sports events. Preparedness and planning should be different, and suited for the patterns of patients. It will be necessary to study more cases to characterize various patterns of patients in a media center. Keywords: demography; football; mass gathering; media center; medical aid; patterns; planning; preparedness; soccer; sports event; World Cup Prehosp Disast Med 2002;17(s2):s85.

\section{Medical Emergency Center of Wuhan, China Yue An Xiong \\ Director of the Medical Emergency Center of Wuhan, Hubei, China}

Wuhan is a center of industry, finance, business, science, culture, education, and health in the middle of China. It has 8.13 million people in an area of $8,467 \mathrm{sq} \mathrm{km}$. It is called the River City because both the Yangtze and the Han rivers run through it. Wuhan is a vital point of land 